\title{
Treatment of cervical cancer: the importance of a multidisciplinary team approach
}

\author{
J. Alejandro Pérez Fidalgo • Ana Hernández Machancoses • Víctor Martín González • Andrés Cervantes
}

$\mathrm{C}$ ervical cancer is a worldwide major concern, as it is the second most common malignancy in women and a major cause of morbidity and mortality in developing countries. Management of patients with cervical cancer is a complex issue but close collaboration among clinicians of different disciplines can achieve long-term survival in $70 \%$ of patients. Surgery is the most accepted strategy for early cervical cancer and chemoradiation is the standard approach for more advanced stages (IIB-IIIA-IIIB-IVAIVB). However, the optimal approach for stages IB2-IIA is somewhat controversial and different sequential strategies have been explored in this setting. This editorial aims to address several important questions in the therapeutic planning of this disease.

\section{What is the standard approach for early cervical cancer?}

\section{Concurrent chemoradiation}

In 1997 Landoni et al. [1] evaluated the role of radiotherapy alone vs. surgery in stages IB-IIA. Radiotherapy demonstrated significantly less severe morbidity vs. surgery $(12 \%$

J.A. Pérez Fidalgo (区) · A. Cervantes

Servicio de Hematología y Oncología Médica

INCLIVA, Hospital Clínico, Universidad de Valencia

Avda. Vicente Blasco Ibáñez, 17

ES-46010 Valencia, Spain

e-mail: japfidalgo@msn.com

A. Hernández Machancoses

Servicio de Radioterapia

Hospital General Universitario de Valencia

Valencia, Spain

V. Martín González

Servicio de Ginecología

INCLIVA, Hospital Clínico, Universidad de Valencia

Valencia, Spain vs. $28 \%$ ) with identical survival. Moreover, in patients with stage IB2 treated with surgery, adjuvant radiotherapy was required in $84 \%$ due to high-risk features.

Several trials evaluating the addition of chemotherapy with both platinum and non-platinum regimens demonstrated a benefit vs. radiotherapy alone in locally advanced cervical cancer. A metanalysis with 4921 patients from 24 randomised trials confirmed that concurrent chemoradiation is superior to radiotherapy alone. Chemoradiotherapy with both platinum and non-platinum agents improved disease-free and overall survival and reduced both local and distant recurrences [2]. A second metanalysis limited to studies assessing efficacy of cisplatin-based regimens concurrent with radiotherapy confirmed a benefit in overall survival with a reduction in the relative risk of death by $26 \%$ favouring concomitant cisplatin [3].

Concurrent chemoradiation not only prolonged survival but may have achieved pelvic control rates up to $90 \%$. Thus, treatment with concurrent chemoradiation is the current standard of care in Europe for stages IB2-IVA [4]. This strategy was followed by Reig et al. [5], in this retrospective analysis of 56 patients stage IB2-IV treated with external radiation with concurrent weekly cisplatin followed by brachytherapy. With a proportion of stage IIIIV being $14.4 \%$, median survival at 5 years was $80.4 \%$ and pelvic control rate was $71.5 \%$ in this study.

New techniques like extended field intensity-modulated radiation therapy (IMRT) have the potential to improve the therapeutic ratio because of its ability to escalate dose to cancer targets while sparing adjacent healthy tissue [6]. Several retrospective series of patients treated with IMRT claimed dosimetric and clinical benefits, with reduction in acute toxicity compared with historic controls [7].

Another recent improvement in the planification and staging of radiation therapy is the use of PET/CT. By providing metabolic imaging information, this technique has demonstrated a high accuracy $(85.1 \%)$ and specificity $(92.6 \%)$ for predicting lymph node involvement [8], confirming its value in locoregional staging. PET-CT could be used as a tool in the delineation of tumour volumes and the preparation of patient treatment plans, especially when integrated with virtual simulation [9]. 
However excellence in chemoradiotherapy might be difficult to export to the community and results are difficult to reproduce in non-experienced centres. Studies performed in the USA demonstrated that radiation facilities treating fewer than 500 patients per year (total patients including all pathologies) more frequently administered lower total dose, and had lower brachytherapy rates, more frequent prolongation of treatment to more than 70 days and increased indication of adjuvant hysterectomy [10].

Neoadjuvant chemotherapy followed by radiotherapy

In 1998, a metanalysis assessing the role of neoadjuvant CT before RT showed a negative impact on survival when this strategy was used. Several hypotheses tried to explain this observation. Poor patient compliance and toxic deaths due to long chemotherapy regimens are some of the clinical factors that potentially contributed to this result. Accelerated repopulation of tumour-resistant clones might be another important factor. Hence, this strategy was abandoned for the concurrent chemotherapy approach. Similarly a more recently published metanalysis showed a deleterious effect on survival unless a quick dose-dense regimen is used [11].

Neoadjuvant chemotherapy followed by surgery

Neoadjuvant chemotherapy could reduce tumour volume, rendering surgery feasible in inoperable tumours. This approach has been controversial. A metanalysis assessed the efficacy of neoadjuvant CT followed by surgery compared to RT alone. Data from 21 trials showed a significant reduction of the risk of death for the neoadjuvant $\mathrm{CT}$ arm [12]. In another metanalysis of neoadjuvant $\mathrm{CT}$ followed by surgery vs. surgery alone, neoadjuvant $\mathrm{CT}$ prolonged progression-free survival but with no benefit for overall survival [13].

As it remains unclear whether this strategy might be of benefit vs. concurrent chemoradiotherapy, it should be considered only as en experimental approach [14].

\section{What is the benefit of adding adjuvant CT?}

In 2009 Dueñas-González et al. communicated the results of a phase III study addressing this question [15]. This study analysed the effect of adding gemcitabine to cisplatin during the concurrent chemoradiation phase followed by two courses of adjuvant gemcitabine and cisplatin compared to standard chemoradiation with single-agent cisplatin. In both arms brachytherapy was administered after completion of chemoradiation; 515 patients stages IIB/IIIB/IVA were included. The gemcitabine arm showed an improved progression-free survival at 3 years $(74 \%$ vs. $65 \%$ ) and overall survival (78\% vs. $69 \%, p=0.0029$ ). Survival outcome of the control arm was consistent with previous studies, suggesting that the benefit was not due to undertreatment in the chemoradiation population. The incidence of grade 3/4 toxicities was significantly higher in the gemcitabine group than in the standard therapy group (86.5\% vs. $46.3 \%)$. However, despite the increased toxicity, most patients were able to complete the planned treatment. It remains unclear whether the benefit observed in survival is explained by the effect of adding gemcitabine to cisplatin during the concurrent chemoradiation or if it is a benefit attributable to the two adjuvant courses of chemotherapy. Despite these encouraging data, until these results are reproduced in other studies, adjuvant chemotherapy is not recommended outside a clinical trial.

\section{Is adjuvant hysterectomy after RT a recommended approach?}

Data about adjuvant hysterectomy after RT are scant. A retrospective institutional experience of the University of Florida found no differences in the rate of pelvic recurrence before and after the adoption of adjuvant hysterectomy [16]. The only phase III prospective randomised trial was conducted by the GOG [17]. In this study, 282 patients with bulky IB stage cervical cancer were recruited over a period of 7 years. Although the rates of grade 3-4 side effects were similar in both arms, the frequency of any reported adverse effect was $63 \%$ in the hysterectomy vs. $56 \%$ in the RT-alone arm. No differences in terms of overall survival were found $(p=0.26)$ between the two strategies. In this study, operated patients were categorised as having no residual, microscopic or macroscopic residual disease and a significant correlation between pathologic findings and survival was observed. Moreover, conservative surgery could be appropriate for patients with small disease confined to the cervix, persistent or recurrent after primary radiation therapy for bulky IB or IIA stage cervical cancer $[18,19]$.

\section{What is the standard treatment for cervical adenocarcinoma?}

Adenocarcinoma is the second most common histology in cervical cancer, comprising $20 \%$ of the cervical cancers [20]. This histology is becoming more and more common nowadays and it has been associated with a poor prognosis and decreased survival. Most clinical trials in cervical cancer include a proportion of adenocarcinomas and therefore the conclusions of these trials are commonly applied to all histological subtypes. However, adenocarcinoma is underrepresented in most trials. As a consequence, our understanding of the natural history and optimal manage- 
ment of adenocarcinoma of the cervix is limited. In the series by Reig et al., $13 \%$ of the patients presented this particular subtype. In the multivariate analysis of this series, non-squamous histologies were a significant negative prognostic factor for 5-year survival, with a lower survival rate by $20 \%$. Previous studies suggested that adenocarcinoma might be more sensitive to chemotherapy or radiotherapy administered in the adjuvant setting after hysterectomy [21]. Therefore, some authors support that surgical treatment might offer better survival for cervical adenocarcinoma. However, the optimal management of adenocarcinoma of the cervix continues to be a subject of debate among practitioners regarding whether or not it should be different from the management of squamous cell carcinoma.

\section{What is the best way to evaluate prognosis: surgical specimen examination vs. new imaging techniques}

Residual disease after radical therapy is considered an important prognostic factor for recurrence and survival. Assessment of the residual disease is a complex issue that varies depending on the therapeutic strategy.

In the GOG trial of RT followed by hysterectomy vs. RT alone, residual disease in the pathologic samples was a strong predictor of recurrence or decreased survival. In a recently reported series [22] of patients treated with concomitant chemoradiation followed by radical hysterectomy, pathologic assessment was identified as an independent prognostic factor for disease-free and overall survival.

However, in patients treated with chemoradiation, where pathologic samples after treatment are not available, new imaging techniques might provide important prognostic data. The role of F-18 fluorodeoxyglucose (FDG)-PET as a predictive factor of recurrence after IMRT [23] and as a prognosis biomarker in the assessment of regional lymph nodes was recently reported [24, 25]. In 452 patients treated with either IMRT or non-IMRT, FDG-PET was performed before initiating the IMRT and 3 months thereafter to evaluate treatment response. The post-therapy PET was correlated with overall recurrence risk $(p<0.0001)$ and cause-specific survival $(p<0.0001)$. Two other studies published by the same group analysed the role of the FDG-PET in lymph nodes before therapy. FDG uptake was identified as a prognostic factor and predictor for response to treatment, pelvic recurrence and survival.

Excellence in the management of cervical cancer is a major challenge for clinicians. The lack of information about prognostic pathological or molecular factors and complexity of local control are major concerns in the treatment of this malignancy. The multidisciplinary approach has been the cornerstone of successful management in the past and it may be foreseen that the role of interdisciplinary collaboration will be even more important in the future.

\section{References}

1. Landoni F, Maneo A, Colombo A et al (1997) Randomised study of radical surgery versus radiotherapy for stage Ib-IIa cervical cancer. Lancet 30:535-540

2. Green J, Kirwan J, Tierney J et al (2005) Concomitant chemotherapy and radiation for uterine cancer of the cervix. Cochrane Database Syst Rev (3):CD002225

3. Lukka H, Hirte H, Fyles A et al (2002) Concurrent cisplatin-based chemotherapy plus radiotherapy for cervical cancer. A meta-analysis. Clin Oncol (R Coll Radiol) 14:203-212

4. Haie-Meder C, Morice P, Castiglione M (2010) Cervical cancer: ESMO clinical recommendations for diagnosis, treatment and follow-up. Ann Oncol 21[Suppl 5]:v37-v40

5. Reig A, Membrive I, Foro P et al (2011) Long term results and prognosis factors of patients with cervical carcinoma treated with concurrent chemoradiotherapy. Clin Transl Oncol 13:504-508

6. Loiselle C, Koh WJ (2010) The emerging use of IMRT for treatment of cervical cancer. J Natl Compr Canc Netw 8:1425-1434

7. Salama JK, Mundt AJ, Roeske J et al (2006) Preliminary outcome and toxicity report of extendedfield, intensity-modulated radiation therapy for gynecologic malignancies. Int $\mathrm{J}$ Radiat Oncol Biol Phys 65:1170-1176

8. Choi HJ, Roh JW, Seo SS et al (2006) Comparison of the accuracy of magnetic resonance and posiron emission tomography/computerized tomography in the presurgical detection of lymph node metastases in patients with uterine cervical carcinoma: a prospective study. Cancer 106:914-922

9. Carola P, Fanti S (2010) PET-CT and radiotherapy in gynecological cancer. Q J Nucl Med Mol Imaging 54:533-542
10. Eifel PJ, Moughan J, Erikson B et al (2004) Patterns of radiotherapy practice for patients with carcinoma of the uterine cervix: a patterns of care study. Int J Radiat Oncol Biol Phys 60:11441153

11. Tierney JF, Vale C, Symonds P (2008) Concomitant and neoadjuvant chemotherapy for cervical cancer. Clin Oncol (R Coll Radiol) 20:401-416

12. Neoadjuvant Chemotherapy for Locally Advanced Cervical Cancer Metanalysis Collaboration (2003) Neoadjuvant chemotherapy for locally advanced cervical cancer: a systematic review and metanalysis of individual patient data from 21 randomized trials. Eur J Cancer 39:2470-2486

13. Rydzewska K, Tierney J, Vale CL et al (2010) Neoadjuvant chemotherapy plus surgery versus surgery for cervical cancer. Cochrane Database Syst Rev CD0007406

14. Al-Manosur Z, Vershraegen C (2010) Locally advanced cervical cancer: what is the standard of care?. Curr Op Oncol 22:503-510

15. Dueñas-González A, Zarba JJ, Alcedo JC et al (2009) A phase III study comparing concurrent gemcitabine (Gem) plus cisplatin (cis) and radiation followed by adjuvant Gem plus Cis versus concurrent $\mathrm{Cis}$ and radiation in patients with stage IIB to IVA carcinoma of the cervix. J Clin Oncol 27:CRA5507

16. Mendenhall WM, McCarty PJ, Morgan LS et al (1991) Stage IB or IIA-B carcinoma of the intact uterine cervix greater than or equal to $6 \mathrm{~cm}$ in diameter: is adjuvant extrafascial hysterectomy beneficial?. Int J Radiat Oncol Biol Phys 21:899904

17. Keys HM, Bundy BN, Stehman FB et al (2003) Radiation therapy with and without extrafascial hysterectomy for bulky stage IB cervical carcinoma: a randomized trial of the Gynecologic Oncology Group. Gynecol Oncol 89:343-353
18. Rutledge S, Carey MS, Pritchard H et al (1994) Conservative surgery for recurrent or persistent carcinoma of the cervix following irradiation: is exenteration always necessary? Gynecol Oncol 52:353-359

19. Maneo A, Landoni F, Cormio G et al (1999) Radical hysterectomy for recurrent or persistent cervical cancer following radiation therapy. Int J Gynecol Cancer 9:295-301

20. Gien LT, Beauchemin MC, Thomas G (2010) Adenocarcinoma: a unique cervical cancer. Gyencol Oncol 118:140-146

21. Peters WA III, Liu PY, Berret II RJ et al (2000) Concurrent chemotherapy and pelvic radiation therapy compared with pelvic radiation therapy as adjuvant therapy after radical surgery in high-risk early-stage cancer of the cervix. J Clin Oncol 18: 1606-1613

22. Ferrandina G, Margariti PA, Smaniotto D et al (2010) Long-term anlysis of clinical outcome and complications in locally advanced cervical cancer patients administered concomitant chemoradiation followed by radical surgery. Gynecol Oncol 119:404-410

23. Kidd ES, Siegel BA, Dehdasti F et al (2010) Clinical outcomes of definitive intensity-modulated radiation therapy with fluorodeoxyglucose-positron emission tomography simulation in patients with locally advanced cervical cancer. Int J Radiat Oncol Biol Phys 77:1085-1091

24. Kidd ES, Siegel BA, Dehdasti F et al (2010) Pelvic lymph node F-18 fluorodeoxyglucos uptacke as a prognostic biomarker in newly diagnosed patients with locally advanced cervical cancer. Cancer 116:1469-1475

25. Kidd ES, Siegel BA, Dehdasti F et al (2010) Lymph node staging by positrón emission tomography in cervical cancer: relationship to prognosis. J Clin Oncol 28:2108-2113 